\title{
Metamaterials for generating space-time coupled few-cycle pulses
}

\author{
A. Zdagkas' ${ }^{1}$ H. Fang ${ }^{1}$, T. Pu ${ }^{1}$, V. Savinov ${ }^{1}$, N. Papasimakis ${ }^{1}$, N. I. Zheludev ${ }^{1,2}$ \\ ${ }^{1}$ Optoelectronics Research Centre \& Centre for Photonic Metamaterials, University of Southampton, SO17 1BJ, \\ United Kingdom \\ ${ }^{2}$ Centre for Disruptive Photonic Technologies \& TPI, SPMS, Nanyang Technological University, Singapore \\ 637371 \\ a.zdagkas@soton.ac.uk
}

\begin{abstract}
Flying Doughnuts are exact propagating solutions of Maxwell equations in the form of single-cycle, space-time non-separable pulses with complex topology of spectrally broadband vortices. We present the experimental generation of Flying Doughnut pulses and discuss their topological and spatiotemporal structure.
\end{abstract}

Flying Donuts (FDs) are exact solutions to Maxwell's equations and are distinguished by a toroidal arrangement of electromagnetic fields exhibiting strong longitudinal field components along the propagation direction (see Fig. 1a) [1]. The spatial dependence of the FD pulse cannot be separated from its temporal dependence, resulting in a spatially varying frequency spectrum (see Fig. 1b). Indeed, the peak of the frequency spectrum varies across the wavefront with prominent higher frequency components close to the centre of the pulse, whereas lower frequency contributions become dominant at the outer regions of the pulse. FD pulses propagate in free-space at the speed of light and can be manipulated similarly to Gaussian pulses. Owing to the toroidal topology and spatiotemporal coupling, FD pulses can interact with matter in unique ways resulting in non-trivial field transformations upon reflection from perfectly conducting and dielectric interfaces and in excitation of toroidal and anapole resonances in dielectric particles.

(a)

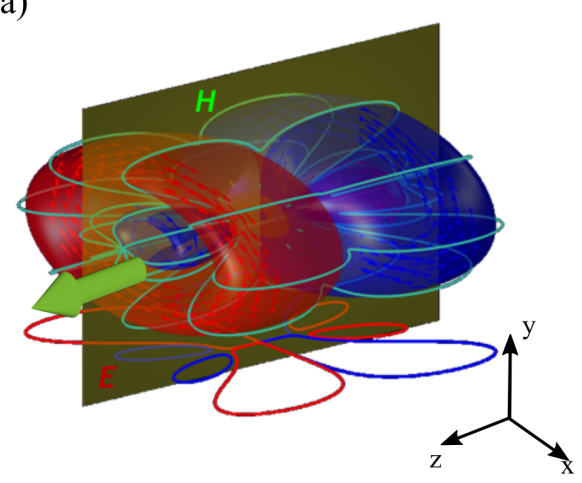

(b)

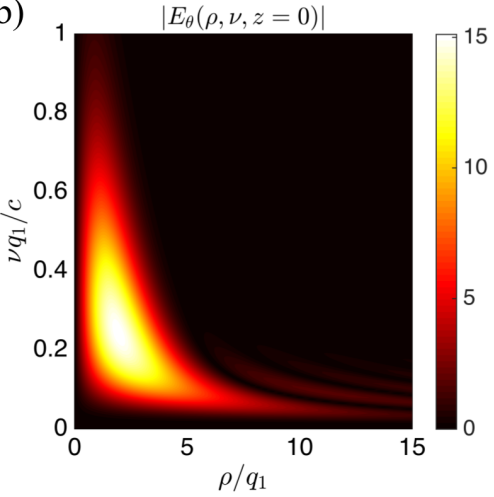

(c)

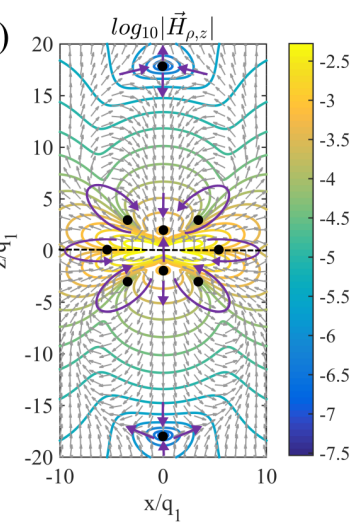

Fig. 1. Spatiotemporal and topological structure of a single-cycle transverse electric (TE) Flying Doughnut pulse at focus: (a) Isosurface of the electric field consisting of two doughnut-shaped regions. Red and blue colours indicate the two half-cycles of the pulse, respectively. Red arrows represent the azimuthally polarized electric field, whereas green lines indicate the direction of the magnetic field winding around the electric field. (b) Spatial variation of the frequency spectrum of the FD pulse with strong high frequency components close to the centre of pulse $(\mathrm{Q}=0)$, whereas lower frequencies dominate the periphery of the array. (c) Isoline and arrow plot of the logarithm of the magnetic field in the xz plane. Black circles indicate the zeros of the magnetic field. Magenta arrows mark the direction of the magnetic field and serve as guide to the eye. In all panels, the pulse propagates along the positive $\mathrm{z}$-axis, while $\mathrm{q}_{1}$ represents its effective wavelength.

The toroidal field configuration of the FD pulse in combination with its space-time non-separable nature results in a surprisingly complex fine-scale structure. In particular, the magnetic field of the pulse vanishes in 10 different regions (black dots in Fig. 1c). Along the propagation axis the magnetic field presents four saddle-like point 
singularities with the longitudinal component pointing towards (away from) and the radial component away from (towards) the singularity. Away from the propagation axis the magnetic field presents six vortex-like singularities. The singularities of the electromagnetic fields are present also in the instantaneous Poynting vector and result in extended regions of energy backflow (not shown here).

The experimental generation of the FD pulses is based on a recently introduced generation scheme, which employs metasurfaces with spatially gradient dispersive response [2]. A schematic of our approach is presented in Fig. 2a, where an incident linearly polarized Gaussian pulse is converted to a Flying Doughnut. In our experiment, a $\sim 10$ fs linearly polarized pulse centered at $800 \mathrm{~nm}$ is incident on a segmented waveplate that converts its polarization to radial. Then, a metasurface comprising concentric plasmonic rings with a width that varies along the radial direction provides the required spatiotemporal coupling (see Fig. 2b). The spatial and temporal profiles of the pulse emerging from the metasurface are characterized through interference with a known reference pulse. Reconstruction of the experimentally characterized generated FD pulse is shown in Fig. 2c. In contrast to the linearly polarized input pulse, the generated pulse is radially polarized according to the toroidal topology of the FD pulse.
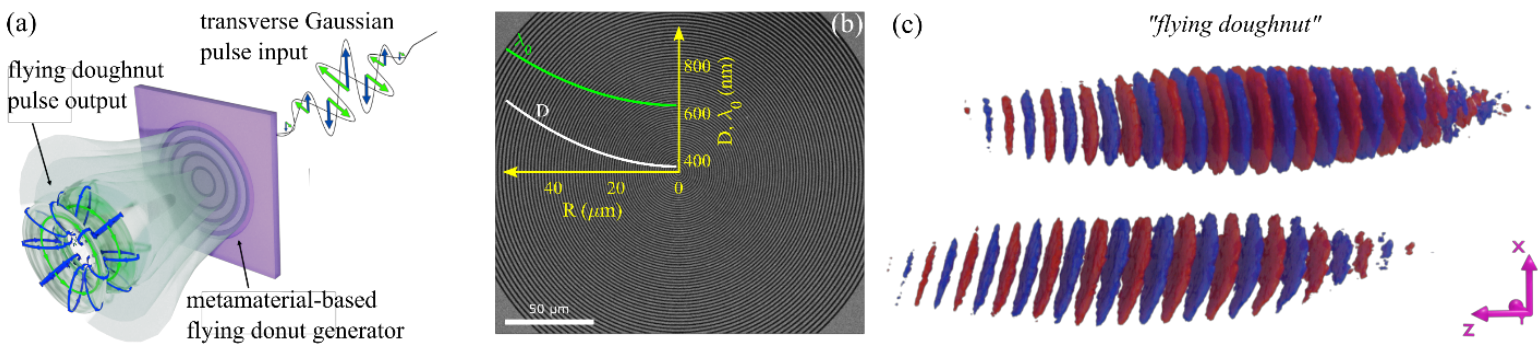

Fig. 2. (a) Pulse generation scheme for Flying Doughnut pulses. A metamaterial-based pulse converter transforms an incident linearly polarized Gaussian pulse to an FD. (b) Scanning electron microscopy picture of the FD generating metasurface. The metasurface is cylindrically symmetric and consists of an array of concentric plasmonic rings with unit cell (white line in inset) increasing from D $250 \mathrm{~nm}$ at the centre of the metasurface to above $600 \mathrm{~nm}$ at the periphery of the array, while the resonance wavelength (green line in inset, $\overline{\lambda_{0}}$ ) shifts from $\sim 600 \mathrm{~nm}$ to $\sim 900 \mathrm{~nm}$. (d) Reconstruction of the experimentally characterized generated pulse propagating along the negative z-axis. Red and blue colours correspond to half-cycle oscillations of the electric field.

In summary, we study the topological properties of FD pulses and demonstrate experimentally their generation in the near-IR range by employing metasurfaces with gradient dispersion properties. We expect that FD pulses will facilitate the study of toroidal and anapole excitations in matter and will find applications in new schemes for information and energy transfer, as well as new forms of spectroscopy.

\section{ACKNOWLEDGEMENT}

The authors acknowledge the support of the Engineering and Physical Sciences Research Council UK, the Singapore Ministry of Education, the Defense Advanced Research Projects Agency (DARPA) Nascent LightMatter Interactions (NLM) Program, and the European Research Council.

\section{REFERENCES}

[1] R. W. Hellwarth and P. Nouchi, "Focused one-cycle electromagnetic pulses," Phys. Rev. E vol. 54, p. 889, 1996.

[2] N. Papasimakis, T. Raybould, V. A. Fedotov, D. P. Tsai, I. Youngs, and N. I. Zheludev, "Pulse generation scheme for flying electromagnetic doughnuts," Phys. Rev. B, vol. 97, p. 201409(R), 2018. 